Gefässchirurgie $2020 \cdot 25: 236-243$

https://doi.org/10.1007/s00772-020-00647-y

Online publiziert: 3 . Juni 2020

(c) Der/die Autor(en) 2020

\section{Wortmann ${ }^{1} \cdot$ K. Meisenbacher' ${ }^{1}$ A. Busch ${ }^{2}$ M. S. Bischoff' $\cdot$ S. Dihlmann' ${ }^{1}$ \\ D. Böckler ${ }^{1}$}

${ }^{1}$ Klinik für Gefäßchirurgie und Endovaskuläre Chirurgie, Universitätsklinikum Heidelberg, Heidelberg Deutschland

${ }^{2}$ Klinik und Poliklinik für Vaskuläre und Endovaskuläre Chirurgie, Klinikum Rechts der Isar, Technische Universität München, München, Deutschland

\title{
Mausmodelle für die Erforschung abdomineller Aortenaneurysmen
}

\section{Warum brauchen wir Mausmodelle zur Erforschung von Aortenaneurysmen?}

Die Entstehung von Aortenaneurysmen ist ein komplexer multifaktorieller Prozess, der bei weitem noch nicht verstanden ist. Die beteiligten pathophysiologischen Prozesse lassen sich grob orientierend in 4 Kategorien einteilen: Degeneration des Bindegewebes der Aortenwand, entzündliche/immunologische Prozesse, genetische Faktoren und biomechanische Effekte (- Abb. 1; [1, 2]).

Die medikamentöse Behandlung von Aortenaneurysmen beschränkt sich momentan weitestgehend auf die Einstellung der kardiovaskulären Risikofaktoren, insbesondere der arteriellen Hypertonie, sowie der Nutzung pleiotroper Statin-Effekte auf Inflammation und PlaqueStabilisierung [3]. Eine kausale medikamentöse Therapie zur Verhinderung des Wachstums oder einer Ruptur ist bislang noch nicht verfügbar.

Humane Gewebeproben, die im Rahmen von offenen Operationen gewonnen werden, sind in diesem Kontext nur eingeschränkt verwertbar, zumal ihre Verfügbarkeit durch die zunehmende Anzahl an endovaskulären Eingriffen eingeschränkt ist. Zusätzlich zeigen die Gewebeproben ausgeprägte inter- und intraindividuelle Unterschiede, die die Interpretierbarkeit stark einschränken. Am schwersten wiegt jedoch die Tatsache, dass Gewebeproben nur aus operationspflichtigen Aortenaneurysmen gewonnen werden können, dem „Endstadium“ der Erkrankung. Eine Aussage über die am Anfang der Erkrankung ablaufenden Prozesse, die wiederum für die Entwicklung potenzieller medikamentöser Therapieansätze entscheiden sind, ist somit nur eingeschränkt oder gar nicht möglich. Darüber hinaus bieten tiermodellbasierte Forschungsansätze eine ideale Grundlage für translationale Forschungsprojekte. Im Mausmodell gewonnene Erkenntnisse können sekundär gezielt an humanen Proben verifiziert werden. Letztlich lässt sich nur so die Relevanz der am Mausmodell erhobenen Befunde in Bezug auf humane Aortenaneurysmen beurteilen.

\section{Übersicht über die vorhandenen Modelle}

Die verfügbaren Mausmodelle für Aortenaneurysmen lassen sich in 3 Kategorien einteilen ( $\bullet$ Tab. 1). Die Entstehung und das Wachstum eines Aortenaneurysmas bis hin zur Ruptur sind multifaktorielle Prozesse mit einer komplexen Interaktion hämodynamischer, physikalischer und inflammatorischer Einflussfaktoren. Wie bei vielen anderen Erkrankungen, deren Pathophysiologien noch nicht endgültig verstanden sind, bildet keines der genannten Modelle das humane Aortenaneurysma komplett ab. Zudem bietet jedes der vorgestellten Modelle individuelle Vor- und Nachteile, sodass die Wahl des Modelles in Abhängigkeit von der zu beantwortenden Fragestellung erfolgen muss. Gegebenenfalls müssen Erkenntnisse, die in einem Mausmodell gewonnen wurden, sogar an einem zweiten Modell nachvollzogen werden, um ausschließen zu können, dass es sich um einen modellspezifischen Effekt handelt.

\section{Medikamentös-systemisch induzierte Modelle}

\section{Angiotensin II-Modell}

Die Induktion von Aortenaneurysmen durch Angiotensin II (Ang II) wurde erstmals 1999 in transgenen Mäusen mit einem Knockout des Low-density-Lipoprotein- (LDL-)Rezeptors gezeigt, wobei die eigentliche Zielsetzung der Experimente die Evaluation des Einflusses von Ang II auf die Entwicklung atherosklero-

Tab. 1 Übersicht über die vorhandenen

Mausmodelle für Aortenaneurysmen. (Mod. nach Daugherty et al. [4])

Genetisch induzierte Modelle

Induktion des Aneurysmas durch Modifikation einzelner Gene

Blotchy-Maus

Lox Knockout

MMP-3/TIMP-1 Knockout

ApoE Knockout

ApoE eNOS Doppel-Knockout

LDL-Rezeptor Knockout

Überexpression von Renin und Angiotensinogen

Chemisch-topisch induzierte Modelle

Lokale Schädigung und der elastischen Fasern der Aortenwand durch eine topische Applikation verschiedener Substanzen

Elastase

Calciumchlorid

Medikamentös-systemisch induzierte Modelle

Systemische Applikation von Substanzen führt zur Ausbildung von Aortenaneurysmen Angiotensin II

LOX Lysyloxidase, MMP Matrixmetalloprotease, TIMP tissue inhibitor of metalloproteinase, ApoE Apolipoprotein E, LDL Lowdensity-Lipoproteine, eNOS endotheliale Stickstoffmonoxid-Synthase 


\section{Degeneration des Bindegewebes der Aortenwand}

Proteolytische Degradierung der Tunica media

Verlust der elastischen Fasern

Verlust der Kollagenstruktur

Überexpression von Matrixmetalloproteasen

- Transition glatter Muskelzellen (VSMCs) von kontraktilem zu synthetischem Phänotyp
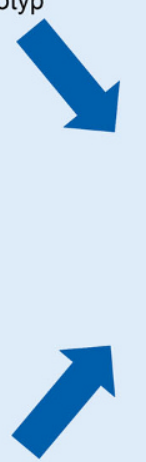

Genetische Faktoren

Marfan-Syndrom

Ehlers-Danlos-Syndrom IV

Familiäre Veranlagung

Einzelnukleotid-Polymorphismen

\section{Entzündliche/immunologische \\ Prozesse \\ Invasion von inflammatorischen Zellen \\ - Lymphozyten \\ - Makrophagen \\ Aktivierung der angeborenen Immunabwehr \\ (z. B. Inflammasome)}
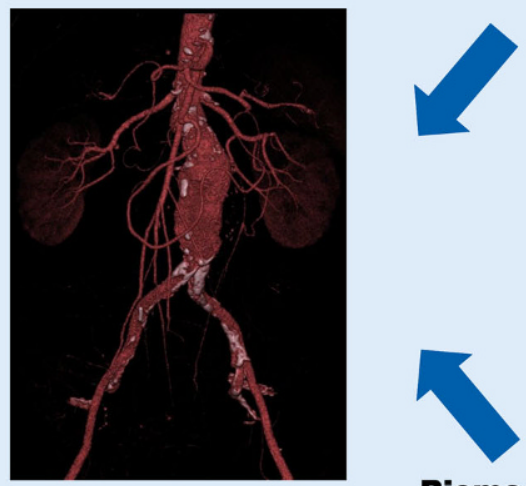

Biomechanische Effekte

- Endothelialer Scherstress

- Turbulenter Blutfluss

- Longitudinaler und orthogonaler Dehnungsstress

- Arterielle Hypertonie

Verlust der Dehnbarkeit der Aortenwand

Abb. 1 ॥ Pathophysiologie des Aortenaneurysmas. Exemplarische Übersicht der Faktoren, die pathophysiologisch zur Entstehung von abdominellen Aortenaneurysmen beitragen [1,27]

tischer Plaques war [5]. Ein Jahr später bestätigte dieselbe Arbeitsgruppe, dass das Modell unter Einsatz von Apolipoprotein E- (ApoE-)Knockout-Mäusen ebenfalls funktioniert [6]. Inzwischen ist bekannt, dass die Inzidenz von Aortenaneurysmen in Mäusen mit Hyperlipidämie, die entweder mittels Fütterung einer fettreichen Diät oder genetisch induziert sein kann, deutlich erhöht ist. Aber auch in Mäusen mit normwertigem Fettstoffwechsel ist eine Induktion von Aneurysmen mittels Ang II möglich, jedoch bei deutlich geringerer Inzidenz (ca. $25-40 \%$ bei Wildtyp(C57Bl/6J-)Mäusen) [7]. Bei ApoE respektive LDL-Knockout-Mäusen beträgt die Inzidenz weit über $60 \%[8,9]$.

Die Applikation von Ang II erfolgt über eine subkutan implantierte osmotische Pumpe, die über einen bestimmten Zeitraum die kontinuierliche Applikation verschiedenster Substanzen erlaubt (- Abb. 2a). Üblicherweise erfolgt eine Therapie mit $1000 \mathrm{ng} / \mathrm{kg}^{\star}$ min über eine Dauer von 28 Tagen. Die osmotischen Pumpen werden in einem gering invasiven Eingriff subkutan im Bereich des Rückens implantiert (• Abb. 2b). Abhängig von der Fragestellung und dem ver- wendeten Mausstamm kann die Dosis und die Dauer der Applikation jedoch variiert werden bis hin zu einer sequenziellen Implantation von mehreren Pumpen [10].

Nach einer rapiden Zunahme des Aortendurchmessers in der ersten Woche folgt anschließend eine langsame kontinuierliche Dilatation der Aorta (• Abb. 2c; [11]). Die Aneurysmen entstehen vor allem im Bereich des Aortenbogens und der suprarenalen Aorta, es können sich jedoch auch langstreckige Aneurysmen der thorakoabdominellen Aorta bilden. Während der ersten beiden Wochen kommt es parallel zu der raschen Expansion zu einer erhöhten Rate an aortalen Rupturen mit oftmals letalem Verlauf (• Abb. 2d). Die Mortalität ist in Abgängigkeit des jeweiligen Versuchsansatzes mit bis zu 25-40\% zu beziffern $[8,11]$.

Passend zu dieser raschen Dilatation in der ersten Phase des Experiments ist der erste Schritt der Aneurysmaformation die Ausbildung einer fokalen Dissektion [12]. Auch die Histologie der Aneurysmen ähnelt eher einer chronischen Dis- sektion mit kompletter Thrombose des Falschkanals (- Abb. 3).

Interessanterweise führt die verwendete Ang-II-Dosierung lediglich zu einem moderaten Blutdruckanstieg von etwa $25 \mathrm{~mm} \mathrm{Hg}$ [13]. Die Entstehung der Aneurysmen scheint also ein substanzspezifischer Effekt von Angiotensin zu sein. Dies kann noch weiter eingegrenzt werden: Während Angiotensin-I-Rezeptor-Blocker die Rate an Aneurysmen signifikant reduzieren, haben Ang-II-Rezeptor-Blocker genau den gegenteiligen Effekt [9].

Zudem ist, ähnlich wie beim Menschen, die Inzidenz bei männlichen Mäusen höher als bei weiblichen [13] und nimmt mit steigendem Alter der eingesetzten Mäuse zu. Unter Berücksichtigung der erhöhten Inzidenz bei Hyperlipidämie spiegelt dieses Modell somit viele epidemiologische Züge humaner Aortenaneurysmen wider.

\section{Chemisch-topisch induzierte Modelle}

Bei den chemisch induzierten Modellen für Aortenaneurysmen wird die Aor- 
Gefässchirurgie 2020 25:236-243 https://doi.org/10.1007/s00772-020-00647-y

(c) Der/die Autor(en) 2020

M. Wortmann · K. Meisenbacher · A. Busch · M. S. Bischoff · S. Dihlmann · D. Böckler Mausmodelle für die Erforschung abdomineller Aortenaneurysmen

\section{Zusammenfassung}

Hintergrund. Mausmodelle zur Erforschung der Pathogenese von Aortenerkrankungen sind seit Jahren in der vaskulären Forschung etabliert. Aufgrund der komplexen multifaktoriellen Prozesse der Entstehung von Aortenaneurysmen, die bis heute noch nicht vollständig verstanden sind, wurden verschiedene Mausmodelle entwickelt. Der vorliegende Artikel stellt eine Übersicht der verfügbaren Modelle sowie der Vor- und Nachteile des jeweiligen Modells vor dem Hintergrund spezifischer Fragestellungen dar. Methodik. Es erfolgte eine selektive Literaturrecherche zum Thema. Ergebnisse. Die verfügbaren Mausmodelle lassen sich in drei Kategorien einteilen: genetisch induzierte, chemisch-topisch induzierte und medikamentös-systemisch induzierte Modelle. Die einzelnen Mausmodelle bieten jeweils spezifische Vor- und Nachteile, sodass die Auswahl des Modelles anhand der zugrunde liegenden Fragestellung erfolgen muss. Idealerweise werden mehrere Modelle kombiniert, um auszuschließen, dass den Ergebnissen modellspezifische Effekte zugrunde liegen. Medikamentös-systemisch induzierte Modelle mittels Angiotensin II und chemisch-topisch induzierte Modelle mittels Kalziumchlorid oder Elastase stellen dabei aufgrund ihrer Flexibilität die am häufigsten verwendeten Mausmodelle dar. Sie erlauben die Untersuchung einer Vielzahl verschiedener Einflussfaktoren oder medikamentöser Therapieansätze.
Schlussfolgerung. Mausmodelle liefern grundlegende Erkenntnisse zur bislang noch unzureichend verstandenen komplexen und multifaktoriellen Pathophysiologie der Entstehung und Progression von Aortenaneurysmen. Tiermodellbasierte Forschungsansätze stellen dabei eine ideale Grundlage für translationale Forschungsprojekte dar, welche prospektiv für die Entwicklung konservativer medikamentöser Therapieansätze für Aortenaneurysmen wesentliche Erkenntnisse beitragen können.

Schlüsselwörter

Mausmodelle · Aortenaneurysma .

Angiotensin II · Kalziumchlorid · Elastase

\section{Murine models for researching abdominal aortic aneurysms}

\section{Abstract}

Background. Mouse models have been established for many years in the research of aortic diseases. Due to the complex multifactorial processes in the pathogenesis of aortic aneurysms, which even today are not completely understood, several murine models have been developed. This review article provides an overview of the available models and discusses the advantages and drawbacks against the background of specific research issues.

Method. A selective literature search was carried out on this topic.

Results. The available murine models can be classified into three categories: genetically induced, chemically induced and druginduced models. The individual murine models each offer specific advantages and disadvantages, so that selection of the model must be based on the underlying research question. Ideally, several models are combined in order to exclude that the results are due to model-specific effects. Drug-induced models with angiotensin II and chemically induced models with calcium chloride or elastase are the most frequently used murine models due to their flexibility. They enable the investigation of a multitude of different influencing factors or drug treatment approaches.
Conclusion. Murine models provide fundamental knowledge on the previously insufficiently understood complex and multifactorial pathophysiology of the formation and progression of aortic aneurysms. Research approaches based on animal models represent an ideal foundation for translational research projects, which can prospectively contribute essential knowledge for the development of conservative drug treatment approaches for aortic aneurysms.

Keywords

Mouse model · Aortic aneurysm · Angiotensin II C Calcium chloride - Elastase tenwand, vor allem die elastischen Fasern, mittels einer topischen Applikation von verschiedenen Substanzen lokal geschädigt. Dies kann entweder mithilfe einer lokalen Perfusion des Gefäßes oder durch Substanzapplikation von außen auf die Aortenwand erfolgen. Durch die Schwächung der Wand kommt es anschließend zur Dilatation des Gefäßabschnitts mit Ausbildung eines Aortenaneurysmas. Hierbei bildet sich initial eine akute Entzündungsreaktion, die anschließend in eine chronische Entzündung übergeht.
Bereits seit 1988 ist bekannt, dass die lokale Applikation von Calciumchlorid $\left(\mathrm{CaCl}_{2}\right)$ auf Gefäße zur Ausbildung von lokalen Aneurysmen führt [14]. Dies wurde 2001 auf die Aorta zur Induktion von Aortenaneurysmen übertragen [15], wobei ein mit $\mathrm{CaCl}_{2}$ getränkter Schwamm lokal auf die infrarenale Aorta aufgebracht wurde. $\mathrm{CaCl}_{2}$ bildet mit den elastischen Fasern Komplexe, die wiederum eine inflammatorische Reaktion mit sukzessiver Destabilisierung und Dilatation der Aortenwand auslösen. 2-10 Wochen nach dieser Intervention bilden sich bei bis zu $100 \%$ der behan- delten Mäuse Aneurysmen aus, die sich bis auf das doppelte des ursprünglichen Durchmessers erweitern.

Eine weitere Substanz zur lokalen Schädigung der Aortenwand mit nachfolgender Aneurysma-Induktion ist Elastase. Die Induktion von Aortenaneurysmen mittels Elastase wurde 1990 erstmals in der Ratte beschrieben [16] und 2000 schließlich auf die Maus übertragen [17]. Initial wurde hierfür ein infrarenaler Abschnitt der Aorta kurzfristig ausgeklemmt, anschließend punktiert und die Elastase über eine Zeit von $5 \mathrm{~min}$ mit einem Druck von $100 \mathrm{~mm} \mathrm{Hg}$ 

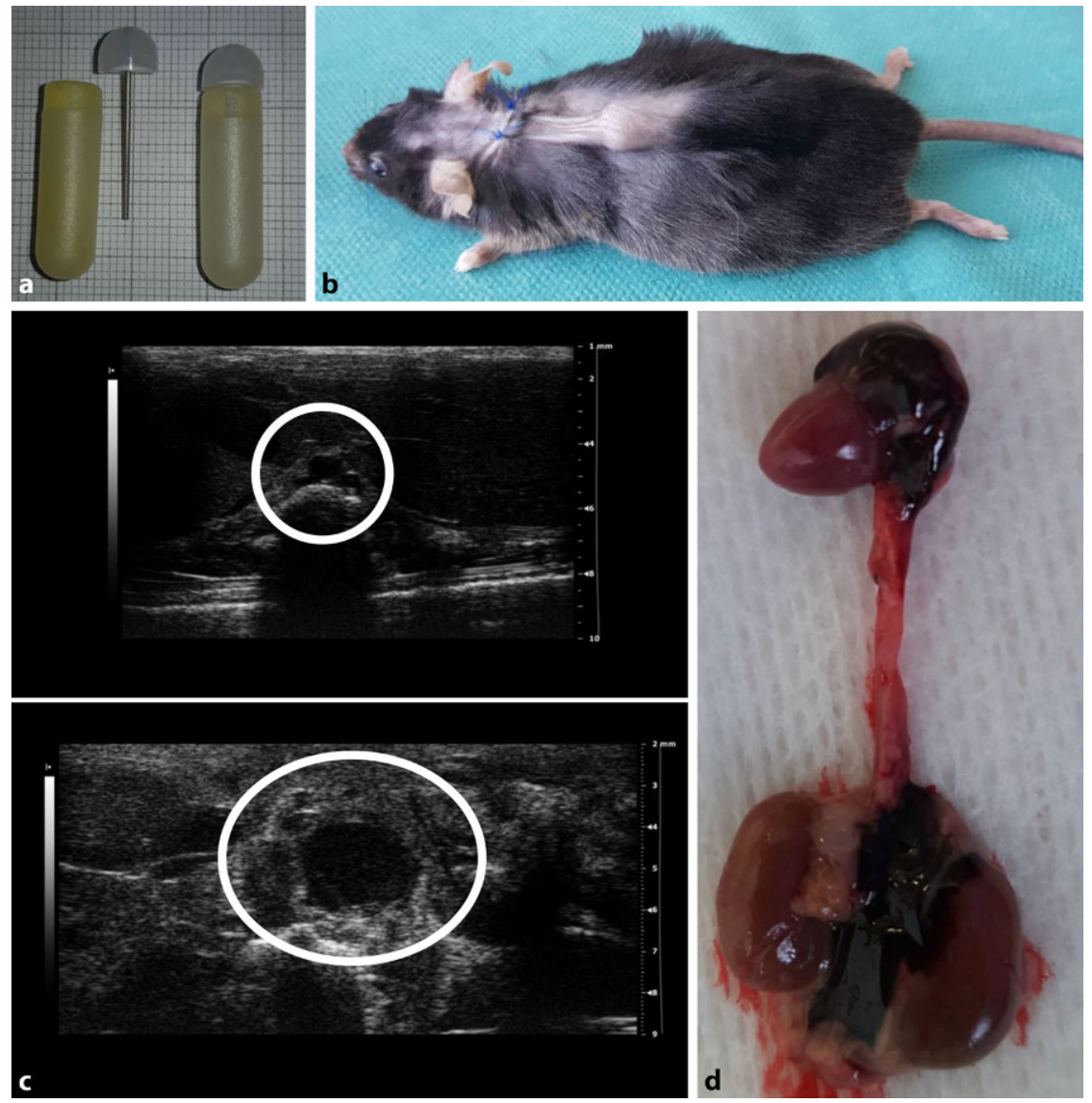

Abb. $2 \Delta$ Angiotensin-II-Mausmodell für Aortenaneurysmen. a Osmotische Pumpe Modell 2004 $\left(\right.$ ALZET $^{\circledR}$, DURECT Corporation, Cupertino, CA, USA), die eine Applikation von insgesamt $200 \mu \mathrm{l} \mathrm{Vo-}$ lumen über einen Zeitraum von 4 Wochen erlaubt. b Subkutan implantierte osmotische Pumpe in eine C57BI/6J-Maus. c Duplexsonographische Darstellung der abdominellen Aorta vor Beginn der Behandlung (oben) und nach Induktion eines Aortenaneurysmas durch Angiotensin-II-Applikation (unten). Die Aorta ist jeweils durch einen Kreis markiert. $\mathbf{d}$ Rupturierte Aneurysmen des Aortenbogens und des suprarenalen Aortenabschnitts durch eine Therapie mit Angiotensin II (1500 ng/kg*min)

in das Gefäß instilliert. Bereits dies führt $\mathrm{zu}$ einer Zunahme des Aortendurchmessers um bis zu $70 \%$ ([17]; • Abb. 4). Allerdings ist dieser Eingriff durch die notwendige Präparation der Aorta, die Punktion, die druckgesteuerte ElastaseApplikation und zuletzt die Übernähung der Punktionsstelle technisch anspruchsvoll. Einfacher ist eine Applikation von Elastase von außen auf die Aorta, welche in über $60 \%$ der Mäuse zur Induktion von Aortenaneurysmen führt [18]. Interessanterweise gibt es jedoch deutliche histologische Unterschiede zwischen intra- und extraluminaler Applikation von Elastase, insbesondere in Bezug auf die auftretende Inflammation [19]. unzureichenden Vernetzung von Elastinund Kollagen-Fasern, da durch den Kupfermangel die Funktion der Lysyloxidase nicht mehr ausreichend gegeben ist. Mit steigendem Alter bilden Blotchy-Mäuse zunehmend Aneurysmen. Die Inzidenz erreicht bei 6 Monaten fast $100 \%$ [20]. Auch ein direkter Knockout der Lysyloxidase führt zur Ausbildung von Aortenaneurysmen [21].

Interessanterweise bilden sowohl ApoE- als auch LDL-Rezeptor-Knockout-Mäuse Aneurysmen unter Fettfütterung aus [22]. Dies verdeutlicht einmal mehr den engen Zusammenhang zwischen Atherosklerose und Aneurysmen. Allerdings ist bei beiden Modellen die Inzidenz von Aneurysmen vergleichsweise gering, sodass die praktische Verwendung zur Erforschung der Pathophysiologie von Aneurysmen eingeschränkt ist.

\section{Kritische Gegenüberstellung der Mausmodelle}

Die beschriebenen genetisch induzierten Modelle sind in ihrer Aussagekraft hinsichtlich der Pathogenese von Aortenaneurysmen deutlich limitiert, da sie vor allem den Effekt eines einzelnen Gens bis hin zu dem durch das entsprechende Gen kontrollierten Signalweg darstellen. Auch die Übertragbarkeit der durch genetische induzierte Modelle gewonnenen Erkenntnisse auf menschliche Patienten bleibt - abhängig von der jeweiligen Fragestellung - limitiert, da Aneurysmen bis auf wenige bekannte Bindegewebserkrankungen multifaktorielle Erkrankungen sind, die sich nicht auf einen singulären Gendefekt zurückführen lassen. Obwohl sie wichtige Erkenntnisse über die Funktion einzelner Proteine geliefert haben, spielen die genetisch induzierten Modelle bei der Erforschung der Pathophysiologie von Aortenaneurysmen nur eine untergeordnete Rolle.

Am weitesten verbreitet sind das Ang II-Mausmodell und die chemischtopisch induzierten Modelle [23]. Vorteil dieser Modelle ist ihre hohe Flexibilität. Der Test von medikamentösen Behandlungen und der Einsatz verschiedener transgener Mauslinien ist meist problemlos möglich, sodass unterschiedlichste 

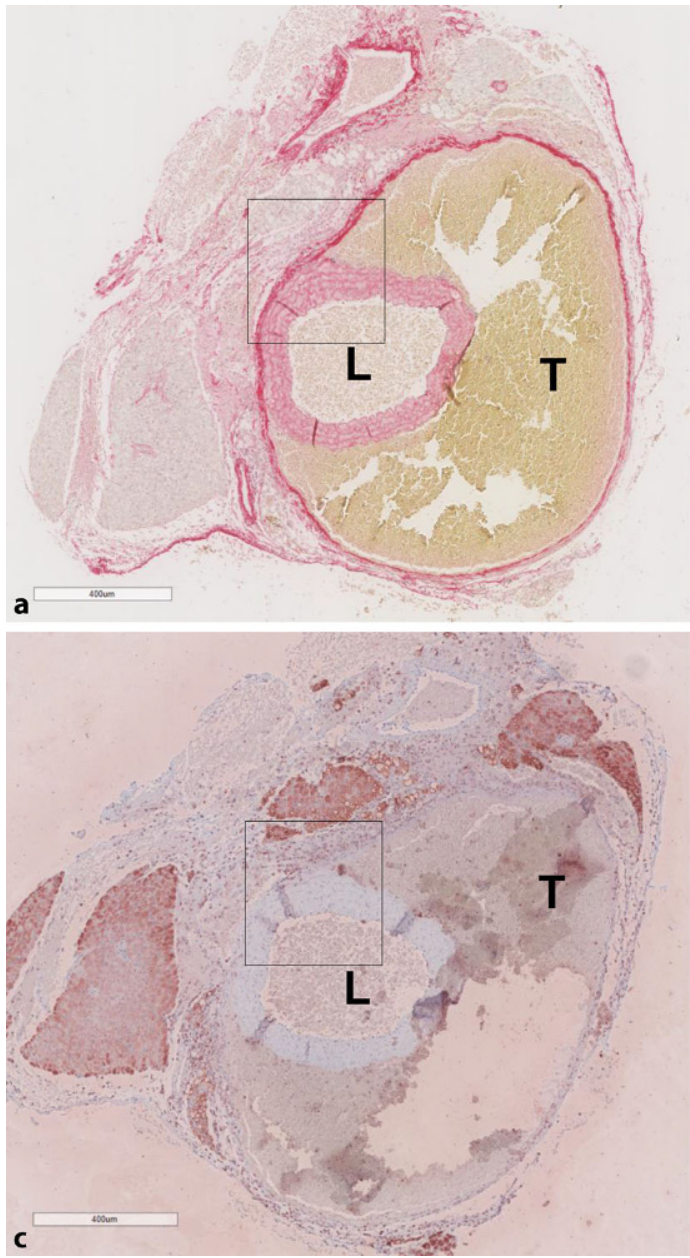
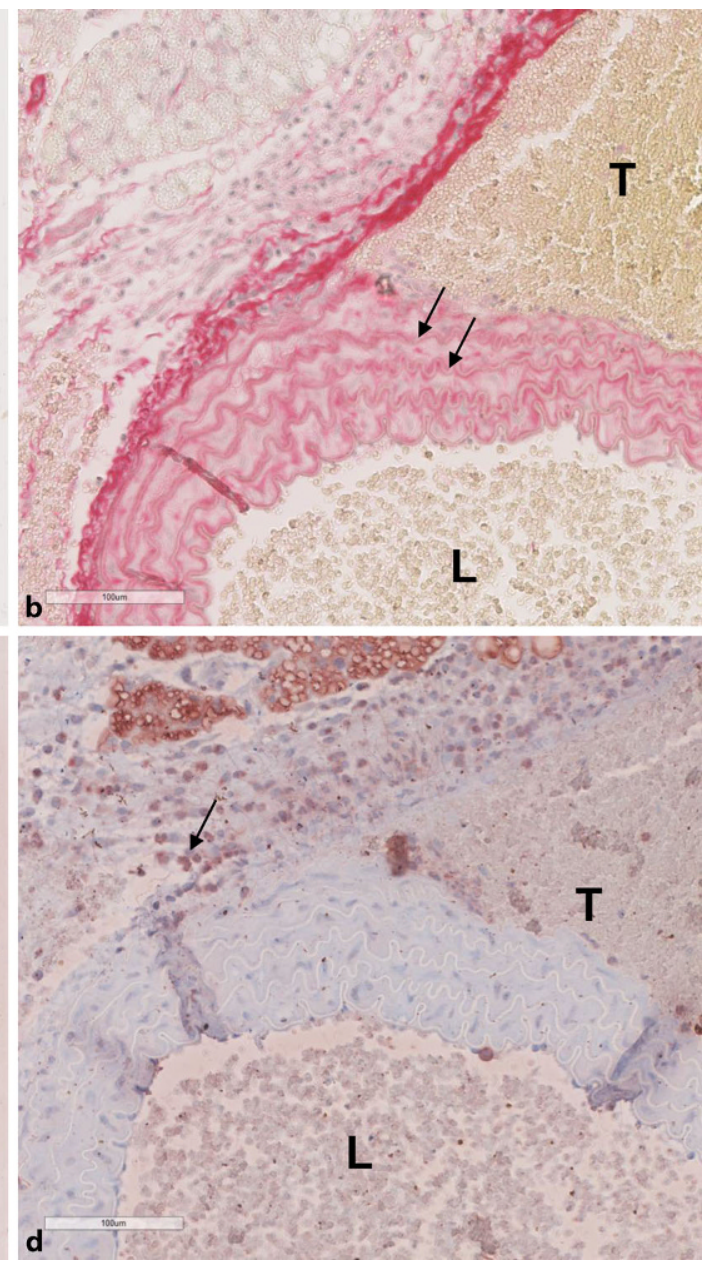

Abb. $3<$ Histologisches Präparat eines Aortenaneurysmas, induziert durch Infusion mit Angiotensin II

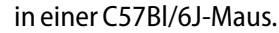
a, b Sirius-Red-Färbung zur Visualisierung von Kollagen und glatten Muskelzellen (rot) a Querschnitt durch das Aneurysma im Bereich des maximalen Durchmessers mit exemplarischer Vergrößerung eines Ausschnitts (b), Pfeile: elastische Fasern. c, d Immunhistochemische Färbung mit einem CD45-spezifischen Antikörper, der die Infiltration mit Leukozyten anzeigt (rotbraun).c Querschnitt durch das Aneurysma im Bereich des maximalen Durchmessers mit exemplarischer Vergrößerung eines Ausschnitts (d) $L$ Lumen, $T$ Thrombus
Fragestellungen beantwortet werden können.

\section{》) Das Ang II Mausmodell und die chemisch-topisch induzierten Modelle sind am weitesten verbreitet}

Bezogen auf die technische Durchführung ist hierbei das Ang II-Mausmodell den chemisch induzierten Modellen überlegen. Die subkutane Implantation der osmotischen Pumpen kann in einer kurzen Narkose mit geringer Belastung für die Versuchstiere und geringer Komplikationsrate durchgeführt werden. Auf eine Laparotomie, wie sie bei den chemisch induzierten Modellen notwendig ist, kann somit verzichtet werden. Die Etablierung dieses Modells erscheint somit vergleichsweise einfach und ist mit einer relativ flachen Lernkurve verbun- den. Erkauft wird dies durch die höheren Kosten, die durch die Verwendung derosmotischen Pumpen zustande kommen.

Der Hauptnachteil des Ang II-Modells ist in der Morphologie der Aneurysmen zu sehen. Während die Aneurysmen sich duplexsonographisch als teilthrombosierte Aortenaneurysmen darstellen, ähneln sie in histologischen Untersuchungen eher Aortendissektionen mit einem vollständig thrombosierten Falschkanal. Wenn man zusätzlich die rasche Entstehung der Aneurysmen in der ersten Woche mit anschließender langsamer Dilatation und die hohe aortenassoziierte Letalität in der Anfangsphase im Hinterkopf behält, ist nicht auszuschließen, dass das Modell eine Mischform zwischen einem Aortenaneurysma und einer Aortendissektion abbildet [12].

Als weiterer Nachteil ist die vergleichsweise hohe Letalität durch die Ang IIApplikation anzuführen. Grund hierfür sind akute Rupturen der Aorta, die vor allem in den ersten beiden Wochen der Ang II-Applikation auftreten. Mortalitätsraten bis zu $40 \%$ machen abhängig vom Endpunkt des Experiments die Kalkulation der Mauszahlen schwierig, können aber auf der anderen Seite einen zusätzlichen Endpunkt im Sinne der aortenassoziierten Letalität bieten.

Mittels $\mathrm{CaCl}_{2}$ induzierte Aneurysmen rupturieren selten und bilden kaum intraluminale Thromben. Da letztere oft eine starke Infiltration mit inflammatorischen Zellen aufweisen und somit eine wichtige Rolle in der Progression von Aortenaneurysmen zu spielen scheinen [24], schränkt dies die Aussagekraft des Modells ein.

Eindeutiger Vorteil dieser Modelle ist, dass man mit hoher Sicherheit an prädestinierten Abschnitten der Aorta Aneurysmen auslösen kann, sei es abdominell oder thorakal ([25]; D Abb. 5). Die Rate an erfolgreichen Induktionen erreicht hierbei nahezu hundert Prozent, sodass 

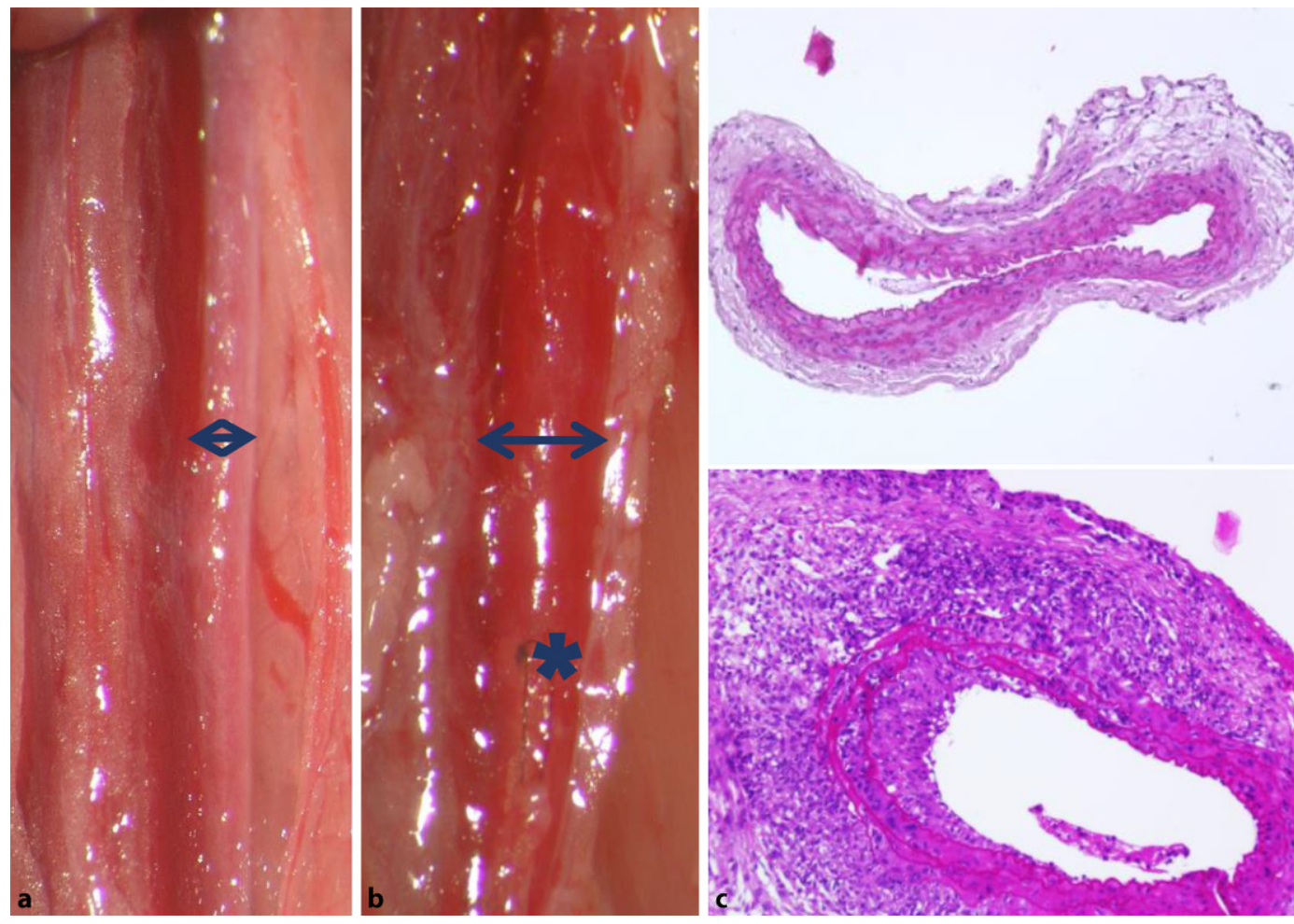

Abb. $4<$ Chemische Induktion von Aneurysmen mittels Elastase. Mausaorta a vor Behandlung und b 14 Tage nach chemischer Induktion eines Aneurysmas mittels intraluminaler Applikation von Elastase. Hierdurch kam es zur Ausbildung eines Aneurysmas mit einem Maximaldurchmesser von $1,2 \mathrm{~mm}(\leftrightarrow)$. Sternchen Übernähung der Punktionsstelle für die intraluminale Elastase-Applikation. c Hämatoxylin-Eosin-Färbung einer unbehandelten Aorta (oberes Bild) und eines mittels Elastase induzierten Aortenaneurysmas (unteres Bild)

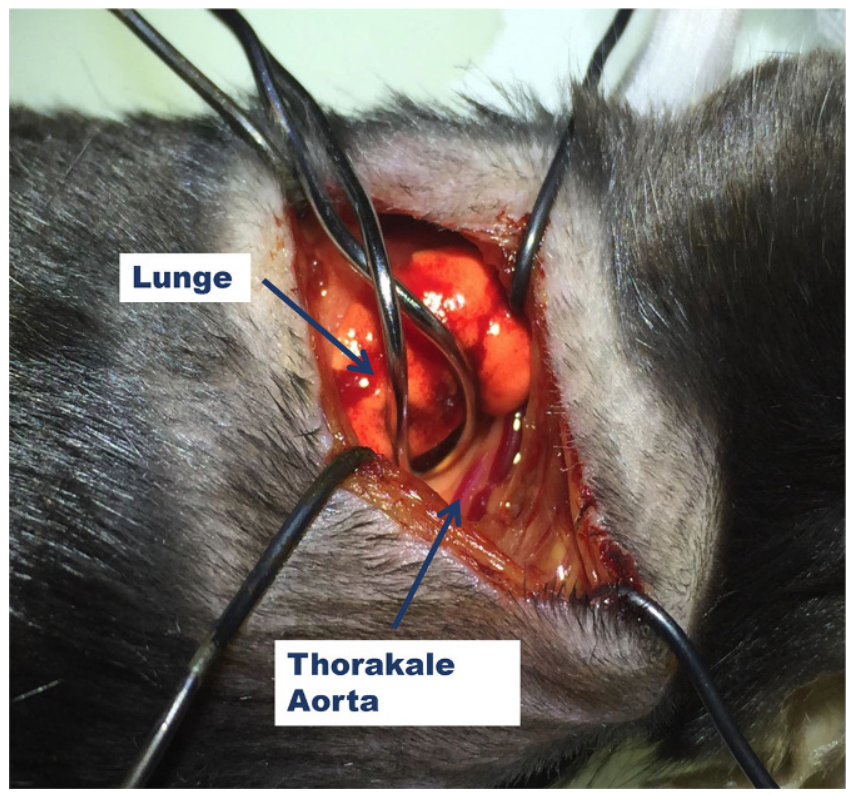

Abb. $5<$ Thorakaler Zugang zur Aorta. Ein Vorteil der chemisch induzierten Mausmodelle ist, dass Aneurysmen an definierten Gefäßabschnitten ausgelöst werden können. Hier gezeigt ist beispielsweise ein Zugang zur thorakalen Aortazur Induktion thorakaler Aortenaneurysmen

die notwendigen Tierzahlen gering gehalten werden können. Außerdem sind die chemisch induzierten Modelle aus finanzieller Sicht vergleichsweise günstig, da lediglich die benötigen Stoffe zur Destabilisierung der Aortenwand benötigt werden.

Im Vergleich zum $\mathrm{CaCl}_{2}$-Modell ist die intraluminale Applikation von Elastase technisch gesehen deutlich aufwendiger, resultiert aber in Aneurysmen, die humane Aortenaneurysmen besser widerzuspiegeln scheinen.

Ob die im Rahmen der durch die chemische Destruktion der elastischen Fasern sowie durch das lokale Gewebetrauma im Rahmen des Eingriffs ausgelöste Entzündungsreaktion mit den in humanen Aneurysmen nachweisbaren in- flammatorischen Prozessen gleichzusetzen sind, ist weitestgehend unklar.

Nachteilig sind darüber hinaus die langsamere Lernkurve der Operateure und die höhere Belastung für die eingesetzten Versuchstiere, die mit der notwendigen Laparotomie mit Freipräparation der Aorta einhergehen.

\section{》) Chemisch-topisch induzierte Aneurysmen können an beliebigen Gefäßabschnitten ausgelöst werden}

Wie bereits mehrfach erwähnt, bildet keines der vorgestellten Mausmodelle das humane Aortenaneurysma in allen seinen Krankheitszügen ab. Aus diesem Grund wurden im Laufe der letzten Jahre die einzelnen Modelle weiter verfeinert, was jedoch die Komplexität auch weiter erhöht. Durch die zusätzliche Gabe eines gegen TGF- (transforming growth factor-) $\beta$ gerichteten Antikörpers während der Induktion von Aortenaneurysmen mittels Elastase kann zum Beispiel ein vermehrtes Wachstum des Aneurysmas bis hin zur Ruptur mit einer verstärkten Entzündungsreaktion der Aorten- 
wand und Bildung eines intraluminalen Thrombus erreicht werden, sodass mehrere distinktive Elemente der humanen Erkrankung besser abgebildet werden [26].

\section{Fazit für die Praxis}

- Mausmodelle liefern grundlegende Erkenntnisse zur bislang noch unzureichend verstandenen komplexen und multifaktoriellen Pathophysiologie der Entstehung und Progression von Aortenaneurysmen. Zudem tragen Sie zur Entwicklung konservativer medikamentöser Therapieansätze bei.

- Die einzelnen Mausmodelle bieten jeweils spezifische Vor- und Nachteile, sodass die Auswahl des Modelles anhand der zu beantwortenden Forschungsfrage erfolgen muss.

- Prinzipiell lassen sich die vorhadenen Modelle in medikamentössystemisch, chemisch-topisch und genetisch induzierte Modelle einteilen.

- Das Angiotensin II und die mittels Calciumchlorid oder Elastase chemischtopisch induzierten AneurysmaModelle sind die häufigsten verwendeten Mausmodelle. Sie erlauben die Untersuchung einer Vielzahl verschiedener Einflussfaktoren oder medikamentöser Therapieansätze.

- Genetisch induzierte Modelle erlauben die genaue Untersuchung des Effekts eines einzelnen Gens respektive des entsprechenden Signalwegs, sind aber im Umkehrschluss auf das jeweilige Gen limitiert und somit wenig flexibel.

\section{Korrespondenzadresse}

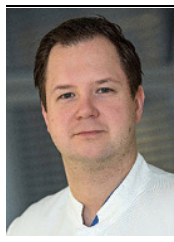

\section{Dr. M. Wortmann}

Klinik für Gefäßchirurgie und Endovaskuläre Chirurgie, Universitätsklinikum Heidelberg Im Neuenheimer Feld 110, 69120 Heidelberg, Deutschland markus.wortmann@ med.uni-heidelberg.de

\section{Einhaltung ethischer Richtlinien}

Interessenkonflikt. M. Wortmann, K. Meisenbacher, A. Busch, M.S. Bischoff, S. Dihlmann und D. Böckler geben an, dass kein Interessenkonflikt besteht.

Für diesen Beitrag wurden von den Autoren keine Studien an Menschen oder Tieren durchgeführt. Für die aufgeführten Studien gelten die jeweils dort angegebenen ethischen Richtlinien.

Open Access. Dieser Artikel wird unter der Creative Commons Namensnennung 4.0 International Lizenz veröffentlicht, welche die Nutzung, Vervielfältigung, Bearbeitung, Verbreitung und Wiedergabe in jeglichem Medium und Format erlaubt, sofern Sie den/die ursprünglichen Autor(en) und die Quelle ordnungsgemäß nennen, einen Link zur Creative Commons Lizenz beifügen und angeben, ob Änderungen vorgenommen wurden.

Die in diesem Artikel enthaltenen Bilder und sonstiges Drittmaterial unterliegen ebenfalls der genannten Creative Commons Lizenz, sofern sich aus der Abbildungslegende nichts anderes ergibt. Sofern das betreffende Material nicht unter der genannten Creative Commons Lizenz steht und die betreffende Handlung nicht nach gesetzlichen Vorschriften erlaubt ist, ist für die oben aufgeführten Weiterverwendungen des Materials die Einwilligung des jeweiligen Rechteinhabers einzuholen.

Weitere Details zur Lizenz entnehmen Sie bitte der Lizenzinformation auf http://creativecommons.org/ licenses/by/4.0/deed.de.

\section{Literatur}

1. Wassef M, Baxter BT, Chisholm RL et al (2001) Pathogenesis of abdominal aortic aneurysms: a multidisciplinary research program supported by the national heart, lung, and blood institute. JVasc Surg 34:730-738

2. Kuivaniemi H, Ryer EJ, Elmore JR et al (2015) Understanding the pathogenesis of abdominal aortic aneurysms. Expert Rev Cardiovasc Ther 13:975-987

3. Salata K, Syed M, Hussain MA et al (2018) Statins reduce abdominal aortic aneurysm growth, rupture, and perioperative mortality: a systematic review and meta-analysis. J Am Heart Assoc 7:e8657

4. Daugherty A, Cassis LA (2004) Mouse models of abdominal aortic aneurysms. Arterioscler Thromb Vasc Biol 24:429-434

5. Daugherty A, Cassis L (1999) Chronic angiotensin II infusion promotes atherogenesis in low density lipoprotein receptor-/- mice. Annals NY Acad Sci 892:108-118

6. Daugherty A, Manning MW, Cassis LA (2000) Angiotensin II promotes atherosclerotic lesions and aneurysms in apolipoprotein E-deficient mice. JClin Invest 105:1605-1612

7. Deng GG, Martin-McNulty B, Sukovich DA et al (2003) Urokinase-type plasminogen activator plays a critical role in angiotensin II-induced abdominal aortic aneurysm. Circ Res 92:510-517

8. Cao RY, Amand T, Ford MD et al (2010) The murine angiotensin Il-induced abdominal aortic aneurysm model: rupture risk and inflammatory progression patterns. Front Pharmacol 1:9
9. Daugherty A, Manning MW, Cassis LA (2001) Antagonism of AT2 receptors augments angiotensin Il-induced abdominal aortic aneurysms and atherosclerosis. Br JPharmacol 134:865-870

10. Rateri DL, Howatt DA, Moorleghen JJ et al (2011) Prolonged infusion of angiotensin II in apoE(-/-) mice promotes macrophage recruitment with continued expansion of abdominal aortic aneurysm. Am J Pathol 179:1542-1548

11. Barisione C, Charnigo R, Howatt DA et al (2006) Rapid dilation of the abdominal aorta during infusion of angiotensin II detected by noninvasive high-frequency ultrasonography. J Vasc Surg 44:372-376

12. Saraff K, Babamusta F, Cassis LA et al (2003) Aortic dissection precedes formation of aneurysms and atherosclerosis in angiotensin II-infused, apolipoprotein E-deficient mice. Arterioscler Thromb Vasc Biol 23:1621-1626

13. Manning MW, Cassi LA, Huang J et al (2002) Abdominal aortic aneurysms: fresh insights from a novel animal model of the disease. Vasc Med 7:45-54

14. Gertz SD, Kurgan A, Eisenberg D (1988) Aneurysm of the rabbit common carotid artery induced by periarterial application of calcium chloride in vivo. JClin Invest 81:649-656

15. Chiou AC, Chiu B, Pearce WH (2001) Murine aortic aneurysm produced by periarterial application of calcium chloride. J Surg Res 99:371-376

16. Anidjar S, Osborne-Pellegrin M, Coutard M et al (1992) Arterial hypertension and aneurysmal dilatation. Kidney Int Suppl 37:S61-66

17. Pyo R, Lee JK, Shipley JM et al (2000) Targeted gene disruption of matrix metalloproteinase- 9 (gelatinase B) suppresses development of experimental abdominal aortic aneurysms. J Clin Invest 105:1641-1649

18. Bhamidipati CM, Mehta GS, Lu G et al (2012) Development of a novel murine model of aortic aneurysms using peri-adventitial elastase. Surgery 152:238-246

19. Busch A, Holm A, Wagner $N$ et al (2016) Extraand intraluminal elastase induce morphologically distinct abdominal aortic aneurysms in mice and thus represent specific subtypes of human disease. JVasc Res 53:49-57

20. Brophy CM, Tilson JE, Braverman IM et al (1988) Age of onset, pattern of distribution, and histology of aneurysm development in a genetically predisposed mouse model. JVasc Surg 8:45-48

21. Maki JM, Rasanen J, Tikkanen H et al (2002) Inactivation of the lysyl oxidase gene lox leads to aortic aneurysms, cardiovascular dysfunction, and perinatal death in mice. Circulation 106:2503-2509

22. Tangirala RK, Rubin EM, Palinski W (1995) Quantitation of atherosclerosis in murine models: correlation between lesions in the aortic origin and in the entire aorta, and differences in the extent of lesions between sexes in LDL receptor-deficient and apolipoprotein E-deficient mice. J Lipid Res 36:2320-2328

23. Lysgaard Poulsen J, Stubbe J, Lindholt JS (2016) Animal models used to explore abdominal aortic aneurysms: a systematic review. Eur J Vasc Endovasc Surg 52:487-499

24. Bhagavan D, Di Achille P, Humphrey JD (2018) Strongly coupled morphological features of aortic aneurysms drive Intraluminal thrombus. Sci Rep 8:13273

25. Ikonomidis JS, Gibson WC, Gardner J et al (2003) A murine model of thoracic aortic aneurysms. J Surg Res 115:157-163 
26. Lareyre F, Clement M, Raffort J et al (2017) TGFbeta (transforming growth factor-beta) blockade induces a human-like disease in a nondissecting mouse model of abdominal aortic aneurysm. Arterioscler Thromb Vasc Biol 37:2171-2181

27. Grundmann RT (Hrsg) (2011) Etiology, pathogenesis and pathophysiology of aortic aneurysms and aneurysm rupture, Intech open

\section{Expertise von Frauen in der COVID-19-Pandemie einbinden}

Frauen sind stärker als Männer von der COVID-19-Pandemie betroffen. Als Beschäftigte im Gesundheits- und Sozialbereich und in den Familien leisten sie den Großteil der Versorgungsarbeit. Dennoch sind sie als Expertinnen nur zu einem geringen Teil miteinbezogen. Das deutsche Chapter des internationalen Expertinnennnetzwerks „Women in Global Health" fordert daher in einer im Mai veröffentlichten Stellungnahme, Frauen als Expertinnen an Entscheidungen paritätisch zu beteiligen und ihre Erfahrungen und Perspektiven in Forschung und Berichterstattung angemessen abzubilden.

Weltweit sind $75 \%$ der Beschäftigten im Gesundheitswesen Frauen, aber nur $25 \%$ in Führungspositionen. 70\% der Arbeit in den Gesundheits- und Sozialpflegeberufen wird in Deutschland von Frauen verrichtet. In der Gesundheits-, Kranken- und Altenpflege, im Rettungsdienst und der Geburtshilfe ist ihr Anteil sogar noch höher.

In der COVID-19-Pandemie sind Frauen aufgrund ihres großen Anteils am Gesundheitspersonal verstärkt einer Infektionsgefahr ausgesetzt. Aktuell sind rund 52\% der Infizierten Frauen. Rund $75 \%$ des infizierten medizinischen Personals in Deutschland ist weiblich. Viele Berufe, die besonders vom Lockdown betroffen sind, zeichnen sich durch einen hohen Frauenanteil aus: Flugbegleiterinnen, Friseurinnen, Verkäuferinnen, Servicekräfte in Gastronomie und Hotels. Frauen in ohnehin unterbezahlten Berufen müssen nun befürchten, Anstellung und Einkommen zu verlieren.

Obwohl Frauen im Rahmen der COVID-19-Pandemie einen Großteil der Versorgungsarbeit leisten, sind sie als Expertinnen nur zu einem geringen Teil miteinbezogen und erhalten nur eingeschränkt die Möglichkeit, ihr Wissen, ihre Erfahrungen und Perspektiven einzubringen.

In den Medien kommen bisher zu wenige Frauen zu Wort: Bei der Berichterstattung zur COVID-19-Pandemie in großen deutschen Zeitungen liegt der Ratio Männer zu Frauen bei 75:21 (Süddeutsche), 8:3 (ZEIT) und in der Frankfurter Allgemeinen Zeitung bei 9:2 (FAZ). Auch im öffentlich-rechtlichen Fernsehen (Markus Lanz 14:6, Maischberger $4: 2$, heute Journal 11:1) sowie im Deutschlandfunk (70:29) gibt es keine ausreichende Vertretung von Expertinnen.

Da Frauen in der COVID-19-Pandemie jedoch in besonderem Maße betroffen sind, ist es wichtig, dass ihre Erfahrungen, Interessen und Perspektiven ausreichend berücksichtigt werden und sie an Entscheidungen paritätisch beteiligt sind.

Es ist daher unabdingbar, dass in Expert*innengremien und -listen wie der Nationalen Akademie der Wissenschaften Leopoldina Frauen paritätisch vertreten sind und in Medienberichten als Expertinnen miteinbezogen werden. In der Pandemie ist es essentiell, die Situation geschlechtersensibel zu bewerten und eine geschlechtersensible Datensammlung und Forschung zu garantieren. Dafür gibt es in Deutschland ausreichend qualifizierte Frauen, die gerne ihre Perspektive und Expertise in den aktuellen Diskurs einbringen.

Wir fordern:

- $\quad$ Frauen in Beratungs- und Entscheidungsgremien einbinden: Paritätische und interdisziplinäre Besetzung sowie Berücksichtigung der gesellschaftlichen Vielfalt in Expert*innenlisten, Gremien, Kommissionen, Beratungsstäben, Talk-Show Runden und auf Konferenzen.

- Frauen in Gesundheitsberufen und der Pflege stärken: Faire Entlohnung und sichere Arbeitsbedingungen für Gesundheitsfachkräfte. Gleiche Verteilung der Pflegearbeit zwischen allen Geschlechtern und deren finanzielle Anerkennung.

\section{- $\quad$ Geschlechtersensible Daten und} Forschung fördern: Konsequente geschlechter- und diversitätssensible Sammlung von Daten (vor allem in der Gesundheitsberichterstattung). Entscheidungen im Krisenmanagement auf Basis einer differenzierten Datenlage treffen.

Für "Women in Global Health - Germany" Prof. Dr. Dr. (mult.) Ilona Kickbusch Prof. Dr. Sabine Ludwig

Die Stellungnahme ist online abrufbar: https://www.womeningh.org/wgh-germany

Quelle: Women in Global Health 